\title{
BNST specific mGlu5 receptor knockdown regulates sex-dependent expression of negative affect produced by adolescent ethanol exposure and adult stress
}

\author{
Chelsea R. Kasten', Eleanor B. Holmgren ${ }^{1}$, Mollie R. Lerner ${ }^{1}$ and Tiffany A. Wills $\mathbb{B}^{1,2}$
}

\begin{abstract}
Adolescent alcohol use is one of the strongest predictors for the development of an alcohol use disorder (AUD). Notably, this period of risk coincides with the development of affective disorders, which disproportionately impact and drive problematic drinking behavior in women. Stress is a particularly salient factor that drives relapse during periods of abstinence. Previous work in our lab has shown that adolescent intermittent ethanol vapor (AIE) produces sexdependent changes in glutamatergic activity in the bed nucleus of the stria terminalis (BNST) and behavioral outcomes following acute restraint stress in adulthood. In females, AIE disrupts group 1 metabotropic glutamate (mGlu1/5) receptor activity and enhances anhedonia-like behavior. The current study site-specifically knocked down mGlu5 receptors in the BNST of male and female Grm5 ${ }^{\text {loxp }}$ mice, exposed them to AIE, and observed the interaction of AIE and stress on negative affect-like behaviors in adulthood. These negative affect-like behaviors included the novelty-induced hypophagia task following acute restraint stress, open field activity, and contextual fear conditioning. Overall, we replicated our previous findings that AIE enhanced anhedonia-like activity in the novelty-induced hypophagia task in females and fear acquisition in males. The primary effect of BNST-mGlu5 receptor knockdown was that it independently enhanced anhedonia-like activity in females. Correlation analyses revealed that behavior in these paradigms showed poor interdependence. These results indicate that preclinical models of negative affective-like states encompass distinct features that may have independent, clinically relevant mechanisms. Further, modulating mGlu5 receptors is a prospective treatment target for females experiencing anhedonic-like states that make them susceptible to alcohol relapse.
\end{abstract}

\section{Introduction}

Adolescent alcohol use is known as one of the strongest predictors for the development of an alcohol use disorder $(\mathrm{AUD})^{1}$, due in part to persistent neuroadaptations that may follow repeated use $\mathrm{e}^{2,3}$. Alcohol use during this critical time period might increase the risk of developing a stress

Correspondence: Tiffany A. Wills (twills@lsuhsc.edu)

${ }^{1}$ Department of Cell Biology and Anatomy, LSU Health Sciences Center New Orleans, New Orleans, LA, USA

${ }^{2}$ Neuroscience Center of Excellence, LSU Health Sciences Center New Orleans, New Orleans, LA, USA

These authors contributed equally: Chelsea R. Kasten, Eleanor B. Holmgren or anxiety disorder, which are more prevalent in females ${ }^{4,5}$. These disorders fall under the umbrella of "negative affect"; the emotional states that underlie numerous psychiatric disorders and are known to drive excessive alcohol drinking, particularly in women ${ }^{6-8}$. Therefore, adolescent alcohol exposure may produce long-term sex-dependent expression of emotional dysregulation that influences problematic drinking in women.

Many facets of negative affect, such as anxiety, depression, and hypervigilance, can be assessed using preclinical models. A recent review from the Neurobiology of Adolescent Drinking in Adulthood (NADIA) Consortium

\section{(c) The Author(s) 2021}

(c) (i) Open Access This article is licensed under a Creative Commons Attribution 4.0 International License, which permits use, sharing, adaptation, distribution and reproduction c. in any medium or format, as long as you give appropriate credit to the original author(s) and the source, provide a link to the Creative Commons license, and indicate if changes were made. The images or other third party material in this article are included in the article's Creative Commons license, unless indicated otherwise in a credit line to the material. If material is not included in the article's Creative Commons license and your intended use is not permitted by statutory regulation or exceeds the permitted use, you will need to obtain permission directly from the copyright holder. To view a copy of this license, visit http://creativecommons.org/licenses/by/4.0/. 
indicates that adolescent alcohol exposure generally produces long-lasting changes in anxiety-like activity in adulthood in rodents ${ }^{9}$. Adolescent alcohol exposure has also been linked to alterations in despair/depressive-like behavior in adulthood that may be sex-dependent, but these results are not consistent ${ }^{10-16}$. The NADIA Consortium notes that these inconsistencies may be due to the alcohol exposure paradigm and that potential differences between sexes have not been systematically addressed ${ }^{9}$. Further, many studies utilize a limited number of behavioral tasks to observe negative affect-like states, even though negative affect is a multi-dimensional concept ${ }^{17-19}$. Recent work from our lab has demonstrated that adolescent alcohol intermittent ethanol vapor (AIE) results in sex-dependent expression of stress-induced negative affect-like states in adulthood. AIE did not alter basal anxiety- or anhedonic-like activity in the absence of stress. However, AIE-exposed female mice showed enhanced anhedonia-like activity in the novelty-induced hypophagia (NIH) task following restraint stress, whereas AIE-exposed male mice showed increased low-intensity foot shockinduced freezing ${ }^{20}$.

One region known to regulate mood-related behaviors is the sexually dimorphic bed nucleus of the stria terminalis $(\mathrm{BNST})^{21}$. The BNST is a site of convergence for stress- and alcohol-related information ${ }^{7}$, which specifically mediate glutamatergic transmission ${ }^{22-24}$. Notably, AIE produces sex-dependent changes in glutamatergic transmission in the BNST of C57Bl/6J mice during acute withdrawal and in adulthood following stress. In male mice, these signaling changes are mediated by the GluN2B-containing NMDA receptors ${ }^{25}$, whereas in female mice they are mediated by group 1 metabotropic glutamate (mGlu1/5) receptors ${ }^{20}$.

Recent work in our lab has demonstrated that this disrupted mGlu1/5-mediated signaling in the BNST is associated with stress-induced anhedonic states in adulthood following AIE that are present in female, but not male, mice ${ }^{20}$. Preclinical studies have consistently implicated mGlu5 receptors in reducing alcohol intake and regulating alcohol-induced negative affect-like behavior across a diverse array of behavioral paradigms, including despair- and anxiety-like behaviors ${ }^{26}$. Further, human postmortem studies show sexually dimorphic mGlu5 receptor expression in individuals with major depressive disorder (MDD) compared to control samples. In the dorsolateral prefrontal cortex, female MDD patient tissue had increased mGlu5 expression, whereas male MDD patient tissue had decreased mGlu 5 expression ${ }^{27}$. However, increased mGlu5 expression was observed in locus coeruleus neurons of male subjects with $\mathrm{MDD}^{28}$.

mGlu5 receptors may also serve as a biomarker for AUDs and interventions. Genetic variants in the mGluR-eEf2AMPAR pathway, including GRM5, predict alcohol intake in community-based samples ${ }^{29}$. In healthy, low-drinking participants, mGlu 5 receptor availability is positively associated with "feeling high" in response to alcohol ${ }^{30}$. Alcoholdependent individuals show reduced mGlu5 receptor availability that widely recovers over 6 months of abstinence ${ }^{31,32}$, although smoking status may be a contributing variable ${ }^{33}$. Notably, mGlu5 availability is positively correlated with craving alcohol as a negative reinforcer and relapse in alcohol-dependent individuals, but recovery of mGlu5 availability over time is associated with reduced alcohol craving $^{31-33}$. Beyond serving as a biomarker, one enticing aspect of investigating mGlu5 receptors is that they represent a clinically viable target. Current potential therapies have shown minimal adverse outcomes over relatively shortterm treatment periods ${ }^{34-36}$, acute alcohol does not affect compound metabolism $^{37}$, and a number of clinical trials have recently wrapped up or are currently recruiting participants to observe alcohol-associated outcomes ${ }^{38-41}$.

The current study sought to identify the role of BNSTspecific regulation of mGlu5 receptors in negative affectivelike behaviors. Using a Cre-lox strategy for the mGlu5 receptor gene, mGlu5 receptors were site-specifically knocked down in the dorsolateral BNST (dlBNST) of male and female mice. The mice were then exposed to AIE and allowed to age into adulthood, at which time anhedonia-like activity in the NIH task following restraint stress, basal anxiety-like activity in the open field, and foot shockinduced freezing were observed. We hypothesized that the AIE GFP-control mice would show the previously observed sex-dependent phenotypes ${ }^{20}$, and that mGlu5 receptor knockdown in the BNST would modulate this behavior in female mice. Elucidating the role of mGlu5 receptors in these behavioral phenotypes may lead to more effective approaches for treating AUDs and comorbid, sex-dependent negative affect-like symptomology.

\section{Methods \\ Mice}

Grm5 $5^{\text {loxp }}$ breeder pairs were generously provided by Dr. Danny Winder (Vanderbilt University, Nashville, TN; Jackson Laboratories, Bar Harbor, ME: B6.129Grm5 $\left.^{\text {tm1.11ixu }} / \mathrm{J}\right)$ and bred in-house. Mice were weaned at PND21 and housed separately with littermates $(2-5$ per cage). Food and water were provided ad libitum. All procedures were approved by the Animal Care and Use Committee at Louisiana State University Health Sciences Center and followed the guidelines set forth by the National Institutes of Health ${ }^{42}$.

\section{Stereotaxic surgery}

A total of 95 mice (51 females, 44 males) received intracranial surgery targeting the dlBNST (AP: +0.14 , L: $\pm 0.8, \mathrm{D}:-4.14)$ between PND22-29. The surgery protocol was modified from Moore and Boehm ${ }^{43}$. Briefly, mice 
were anesthetized using a ketamine/xylazine cocktail $(1 \mathrm{ml}$ of $100 \mathrm{mg} / \mathrm{ml}$ ketamine, $0.1 \mathrm{ml}$ of $100 \mathrm{mg} / \mathrm{ml}$ xylazine, and $8.9 \mathrm{ml}$ sterile saline) at a volume of $0.1 \mathrm{ml} / 10 \mathrm{~g}$ of weight. Mice were then placed on an Angle Two Small Animal Stereotaxic Instrument (Leica Microsystems, Wetzlar, Germany). rAAV5/CamkII-GFP-Cre (cre) or rAAV5-CamKIIa-eGFP (GFP; control) were obtained from the Gene Therapy Center Virus Vector Core Facility (The University of North Carolina at Chapel Hill, Chapel Hill, North Carolina). The virus was infused at a rate of $100 \mathrm{nl} / \mathrm{min}$ for a total volume of $200 \mathrm{nl} / \mathrm{side}$. Injectors were left in place for $5 \mathrm{~min}$ to allow time for the virus to diffuse from the tips. At least one mouse/cage received cre or control virus to reduce potential littermate confounds. Mice received $5 \mathrm{~g} / \mathrm{kg}$ ketoprofen immediately, $24 \mathrm{~h}$, and $48 \mathrm{~h}$ post-surgery and recovered for at least $4 \mathrm{~d}$ prior to vapor exposure. Surgeries for each cohort were completed within a 36-h window.

\section{Western blot}

Viral knockdown was confirmed using western blot for mGlu5 receptors (1:3000; AB5675; Millipore Sigma), normalized to GAPDH signal (1:10,000; AB8245; Abcam, Cambridge, MA), in 500- $\mu \mathrm{m}$ thick dlBNST-containing punches from AIE-naïve adult male and female mice as previously described ${ }^{20}$. Mice were infused with virus during adolescence.

\section{Ethanol vapor exposure}

Six mice died following surgery, leaving 48 females and 41 males to be run through vapor exposure and subsequent behavioral testing. Cages were randomized and assigned to air or ethanol exposure based on sex, vapor cohort, and parents. When possible, at least one cage/sex was run through air and ethanol in each cohort. Male and female mice were exposed in separate chambers that can be independently controlled to produce the same BAC in each sex. The ethanol vapor chambers were maintained to achieve BACs of $\sim 200 \mathrm{mg} / \mathrm{dL}$, which were monitored using nonexperimental mice. Vapor exposure began between PND2735 following previously published protocols ${ }^{25}$. Briefly, mice were placed into the chamber in their home cages overnight for two, 4-d cycles of $16 \mathrm{~h}$ vapor exposure. Mice were placed back in the vivarium for the $8 \mathrm{~h}$ out-of-chamber. The 4-d cycles were separated by $3 \mathrm{~d}$.

\section{Behavioral tasks}

Mice were aged undisturbed until adulthood (PND70 + ). Most mice were run through all behavioral tasks (see Supplemental Table 1 for details).

\section{$\mathrm{NIH}$ and acute restraint stress}

Procedures were modified from previously published protocols $^{44,45}$. Mice were given $2 \mathrm{~h}$ access to Ensure vanilla- flavored nutritional shake in their home cage in a dimly lit room ( 70 lux) under a hood. Latency to consume Ensure was recorded on the second day of training; mice that failed to consume were run through the test portion but excluded from further $\mathrm{NIH}$ analyses. On the test day, mice were restrained in well-ventilated 50-mL conical tubes and left undisturbed for $1 \mathrm{~h}^{46}$ before being returned to their home cage for $1 \mathrm{~h}$. Mice were then individually placed into empty standardized mouse cages under the hood with lights on ( 300 lux) and given $45 \mathrm{~min}$ to consume Ensure. The testing sessions were video recorded and two individuals blind to treatment condition scored the latency to initiate consumption on the test day.

\section{Open field activity}

Three to four weeks later, mice (PND91+) were exposed to the open field arena (TruScan mouse arena, Coulbourn Instruments, $25 \times 25 \mathrm{~cm}$ ) of the contextual fear apparatus located in a sound-attenuated chamber for $3 \mathrm{~min}$ prior to shock administration. This 3 min period was used to assess basal anxiety-like activity, quantified as the $\%$ of total distance traveled in the center of the chamber.

\section{Contextual fear conditioning}

Procedures were modified from a previously published protocol $^{46}$. Following the $3 \mathrm{~min}$ habituation period, mice were exposed to six shocks delivered on a 30 -s variable inter-stimulus interval through the rod shock floor connected to the TruScan Photobeam LINC system. Shocks were $0.4 \mathrm{~mA}, 1 \mathrm{~s}$ duration for male mice and $0.3 \mathrm{~mA}, 500 \mathrm{~ms}$ for female mice. Our lab has previously validated these intensities as producing similar, low levels of freezing in C57Bl6/J mice that allow for the detection of heightened fear responses in AIE-exposed animals $^{20}$. Approximately $24 \mathrm{~h}$ post-training, mice were re-exposed to the chamber for a 15 min extinction session during which no shocks were administered. Data measurements were collected using Tru Scan software on a Windows computer.

\section{Histological confirmation}

Following behavior, mice were initially perfused using 4\% PFA under ketamine/xylazine and targets were checked in $100 \mu \mathrm{m}$ slices. To achieve the best visualization, we switched to sacrificing the mice and using live slices $(200-300 \mu \mathrm{m})$ for ex vivo imaging ${ }^{25}$. The infusion region was visualized by exciting GFP at $488 \mathrm{~nm}$ using a BXWI51 microscope (Olympus). Animals were considered hits when a majority of the fluorescence was located within the dIBNST. Three mice (1 male air-cre, 1 female air-cre, 1 female air-GFP) were removed due to off-target surgeries. 


\section{Statistical analysis}

Only mice with injections determined to target the dIBNST were included in statistical analyses. (Total female mice analyzed: 11 air-GFP, 12 air-cre, 12 AIE-GFP, 11 AIEcre. Total male mice analyzed: 11 air-GFP, 9 air-cre, 11 AIE-GFP, 9 air-cre. Target group sizes were determined by previous findings ${ }^{20}$ ). Male and female mice were analyzed separately due to sex-dependent AIE-induced changes in these behavioral tasks ${ }^{20}$. All data were checked for normality and GraphPad Prism software (ver. 8.3) was used for analyses. Two-way ANOVAs assessing vapor history"viral status were run for the NIH and open field tasks. A threeway ANOVA assessing time point"vapor history"viral status was run for the contextual fear paradigm, as well as two-way ANOVAs within each time point. All values are presented as means \pm SEM with $p \leq 0.05$ considered significant (two-tailed), adjusted for post-hoc analyses. The overall analyses were run once, combining data from 16 litters of mice run in seven distinct cohorts. Sidak's posthoc tests comparing air- vs. AIE-history within viral group were run to confirm replication of the baseline phenotypes previously observed in our $\operatorname{lab}^{20}$.

\section{Results}

Cre infusion significantly knocks down mGlu5 receptors in the dIBNST

The study timeline is shown in Fig. 1A. To validate the flox/cre strategy, mice were infused with cre or control virus at the adolescent timepoint and allowed to age into adulthood without vapor exposure. A t-test indicated that cre infusion targeting the dIBNST significantly reduced mGlu5 receptor expression normalized to GAPDH $(p<$ 0.05 , Fig. 1B, C), which could be visualized by optical excitation of the GFP tag (Fig. 1D).

\section{dIBNST mGlu5 receptor knockdown increases anhedonic- like behavior in female mice}

The NIH task is a preclinical analogue of negative affectassociated despair and anhedonia. Restraint stress uncovers enhanced anhedonic-like behavior in AIE-exposed female, but not male, mice ${ }^{20}$. Further, these stress- and AIEinduced changes in NIH behavior can be modulated by systemic treatment with the mGlu5 receptor antagonist MTEP $^{20}$. To test the role of BNST-specific mGlu5 receptors on this behavior, we site-specifically knocked down these receptors in the BNST. Mice were exposed to AIE, then restraint stress-induced changes in the NIH task were observed in adulthood (Fig. 2A, B).

On the second training day, prior to restraint stress, the latency to consume was measured in the home cage. A vapor history*viral status ANOVA revealed no significant interaction or main effects on latency to consume in female mice ( $p$ 's $>0.05$; Fig. 2Ai). In male mice, a vapor history"viral status ANOVA revealed no significant interaction or main effects on latency to consume ( $p$ 's $>0.05$; Fig. 2Aii), although there was a notable amount of variance in the GFP-infused group. Further, a chi-square test revealed that a significantly higher proportion of female mice failed to consume on the training day $(13 / 46,28.3 \%)$ compared to male mice $(3 / 35,8.6 \%)\left[\chi^{2}(1, N=81)=4.861, p<0.05\right.$; Fig. 2Aiii, iv). These mice were run through the test day but were not included in the test day analyses.

To account for individual and intra-sex variability in consummatory latencies ${ }^{5,47}$, latencies on the test day were quantified as the change in consummatory latency from the training da ${ }^{20}$. In female mice, there was a significant main effect of vapor history $[F(1,29)=4.97, p<0.05]$ and virus $[F(1,29)=7.27, p<0.05]$ (Fig. 3Bi) but no interaction of vapor history"viral status on change in latency to consume on the test day following restraint stress $(p>0.05)$. AIE history and BNST mGlu5 receptor knockdown independently increased latency to consume under aversive conditions. Post-hoc tests revealed no significant effect of AIE-history within virus groups (Sidak $p$ 's $>0.05$ ). In male mice, we observed no significant interaction or main effects of vapor history and virus following restraint stress in the $\mathrm{NIH}$ task ( $p$ 's $>0.05$, Fig. 3Bii). Overall, these results replicate our previous finding that restraint stress induces an anhedonic-like phenotype in adulthood in female mice with an AIE history ${ }^{20}$. Further, mGlu5 knockdown in the BNST enhances this anhedoniclike state in female, but not male mice.

\section{dIBNST mGlu5 receptor knockdown enhances basal anxiety-like activity in males}

Distance traveled in the center of the open field is a wellestablished model of anxiety-like activity ${ }^{48}$. In female mice, a vapor history*viral status ANOVA revealed no significant interaction or main effects ( $p$ 's $>0.05)$ on \% of distance traveled in the center of the open field of the fear chamber in the 3 min prior to shock (Fig. 3A, B).

In male mice, a vapor history"viral status ANOVA revealed no significant effect of vapor history $(p>0.05)$, but a main effect of the virus $[F(1,35)=4.12, p=0.05]$ and a significant interaction $[F(1,31)=5.47, p<0.05]$ (Fig. 3C). Post-hoc analysis indicated that the effect of the virus was driven by the air-history group with mGlu 5 receptor knockdown, with a strong trend towards a significant difference between air-cre and AIE-cre mice (Sidak's; GFP: $p>0.05$, cre: $p=0.052$ ). Regardless of this, air-cre mice showed significantly greater anxiety-like activity than their air-GFP counterparts $(p<0.01)$.

\section{dIBNST mGlu5 receptor knockdown does not alter contextual fear acquisition in either sex but disrupts extinction in female mice}

Freezing behavior in the contextual fear task is considered to be a measurement of hypervigilant states ${ }^{49}$. 


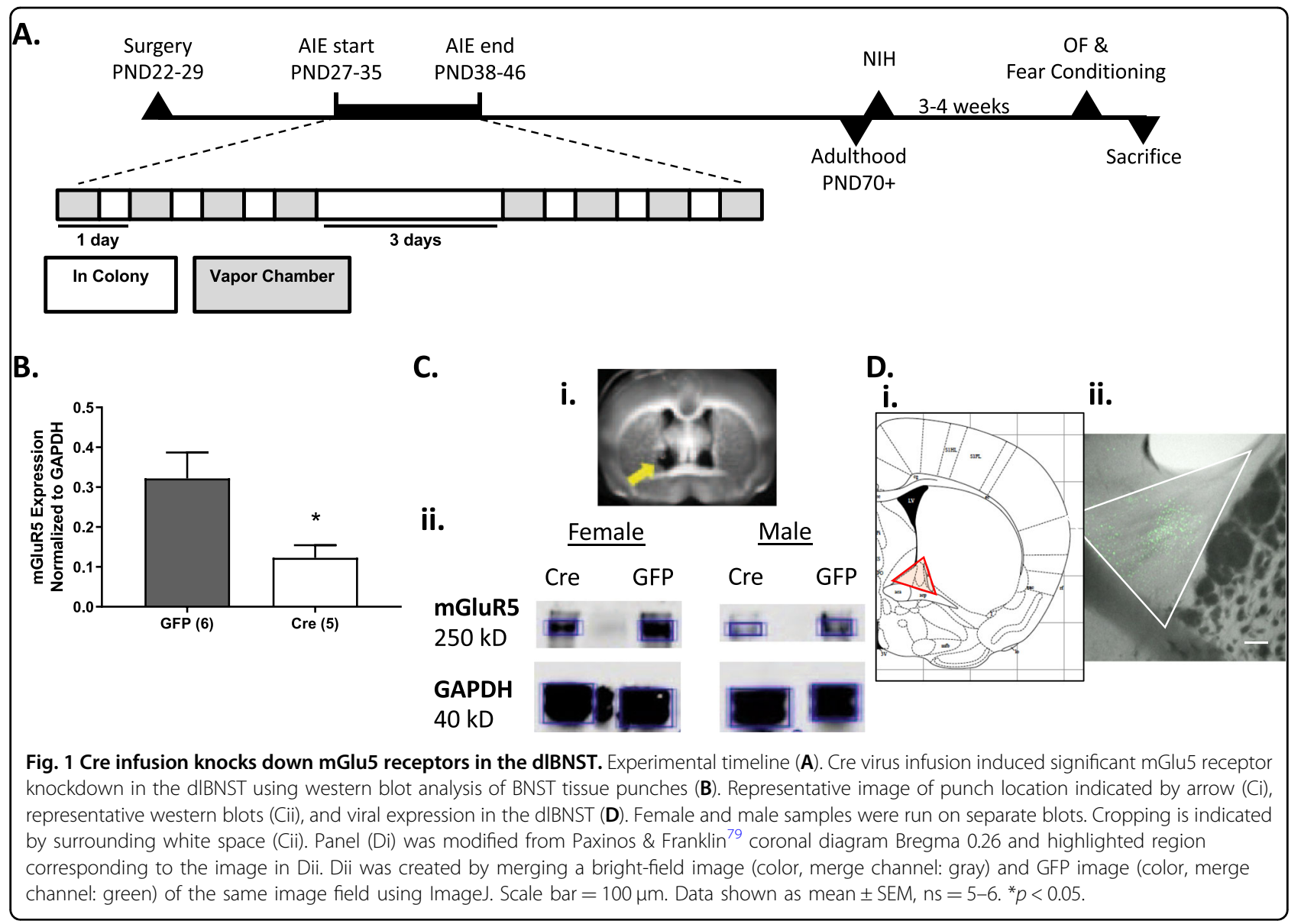

Further, it is regulated by BNST activity ${ }^{50,51}$ and AIE exposure ${ }^{20}$ in male rodents. We induced freezing activity using low-intensity, unpredictable foot shocks ${ }^{20}$ (Fig. 4A). Although a vapor history"viral status ANOVA revealed no significant interaction or main effects $(p$ 's $>0.05)$ on preshock freezing levels in female mice (Fig. 4B), there was a significant interaction effect in male mice $[F(1,35)=4.62$, $p<0.05]$ without any significant main effects or differences between the groups (Fig. 4D). Change scores were used to account for these individual differences in basal freezing levels and intra-sex variability in behavior ${ }^{5,47}$. Freezing at all timepoints was adjusted for each individual mouse's basal pre-shock freezing level (average freezing during the $30 \mathrm{~s}$ immediately preceding the shock). The fear acquisition was quantified as the change in freezing immediately post-shock (30 s), retention of contextual fear memory was quantified as the change in freezing during the initial $1 \mathrm{~min}$ re-exposure to the chamber $24 \mathrm{~h}$ following training, and extinction was quantified as the change in freezing after $8 \mathrm{~min}$ of re-exposure to the chamber with no shock presentation (Fig. 4A). Minute 8 was chosen because freezing levels begin to increase by minute 15 in this low-intensity foot shock task, possibly reflecting habituation to the chamber ${ }^{20}$.
In female mice, a vapor history"viral status*time point ANOVA revealed a main effect of time point $[F(1.92,65.14)=8.81, p<0.001]$. Given our previous findings of time point-specific effects ${ }^{20}$, we also completed vapor history*viral status ANOVAs for each individual time point. We observed no significant interaction or main effects on fear acquisition within the training session or freezing during initial contextual re-exposure ( $p$ 's $>0.05$; Fig. 4C). However, there was a significant main effect of the virus on freezing at extinction minute $8[F(1,37)=5.31$, $p<0.05$ ], where GFP mice showed less freezing (Fig. 4C).

In male mice, a vapor history*viral status"time point ANOVA only revealed a main effect of time $[F(2.00,69.89)=3.15, p<0.05]$. At the post-shock point, a vapor history*viral status ANOVA revealed no significant interaction or main effect of virus $(p$ 's $>0.05)$, but a significant effect of vapor history on fear acquisition within the training session $[F(1,35)=4.98, p<0.05]$ (Fig. 4E). However, Sidak's post-hoc analyses revealed that there was no significant effect of AIE-history within either viral status group ( $p$ 's $>0.05)$. No significant interaction or main effects were observed at the re-exposure or extinction minute 8 timepoints. These results support our previous finding that AIE-history enhances contextual fear acquisition within the 


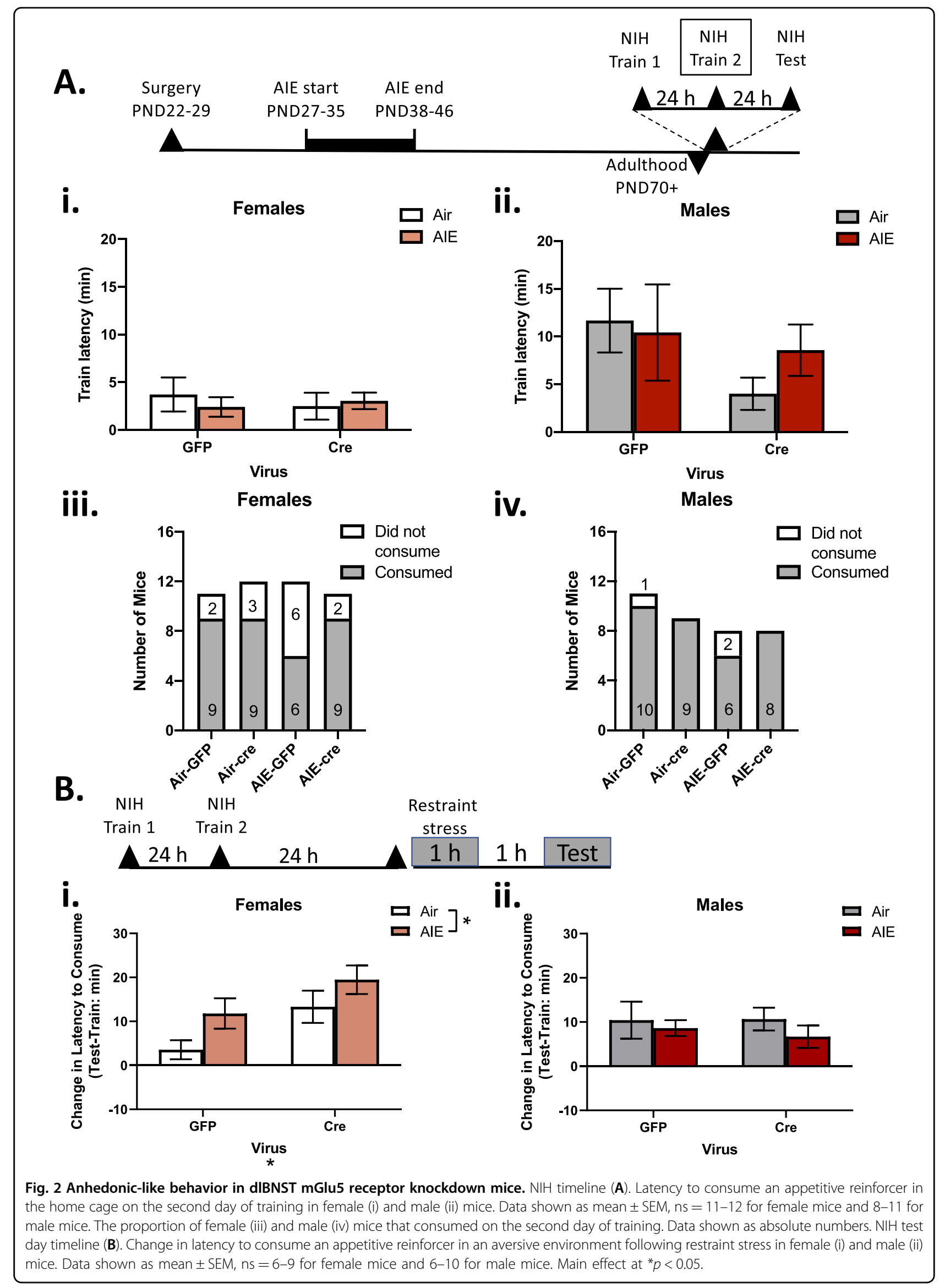




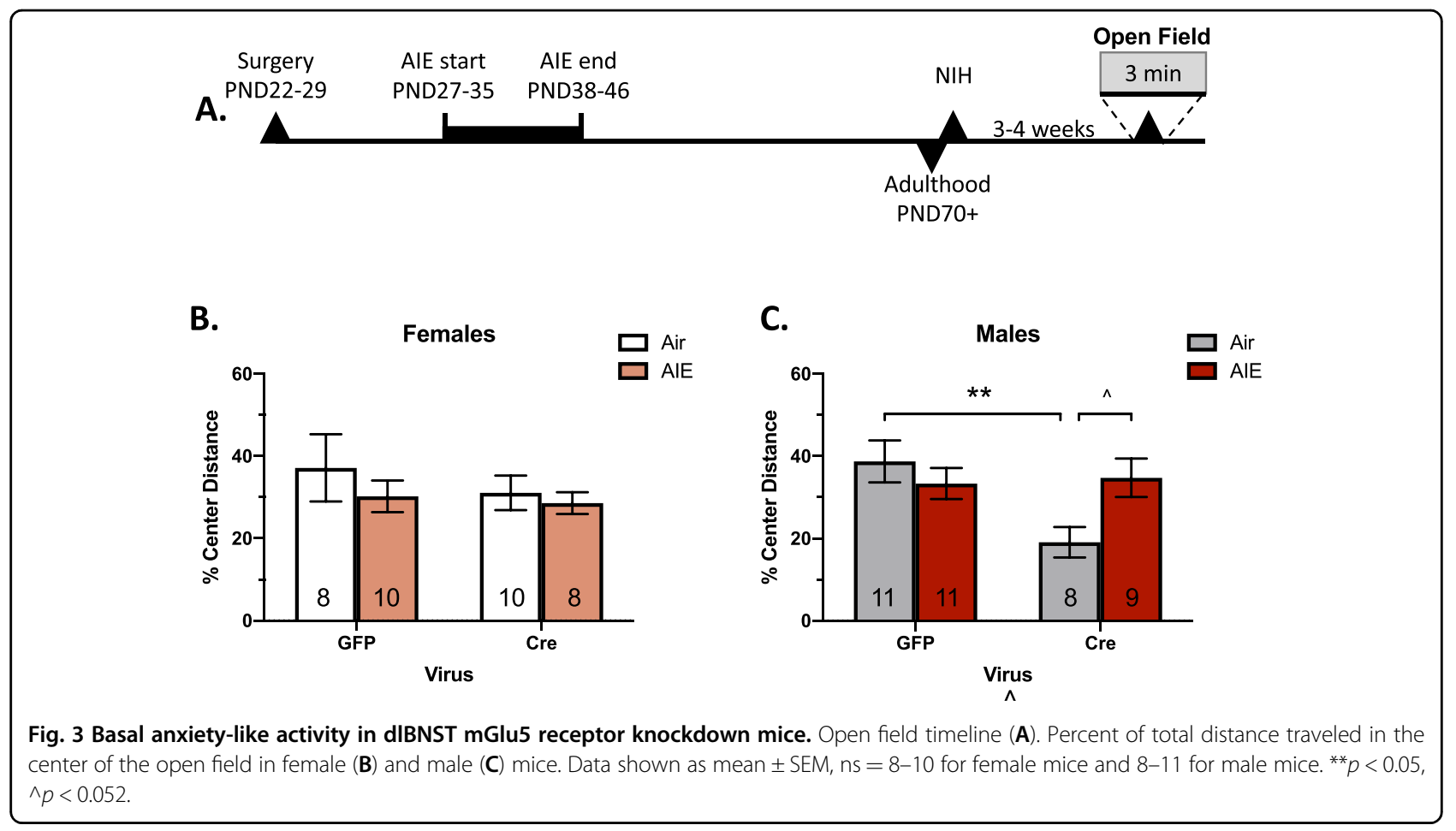

training session in male mice ${ }^{20}$. Notably, this behavior was not affected by mGlu5 receptor knockdown in the BNST.

Negative affect-like facets have minimal interdependency

Negative affective states represent a cluster of symptoms ${ }^{6}$, yet many preclinical studies are unable to investigate the relationship between these symptoms. Because most mice in the current study were run through all behavioral tasks, we were able to assess the individual relationships between basal anxiety (\% center distance, $\mathrm{NIH}$ train latency), anhedonia (NIH test day latency), and hypervigilance (fear acquisition, re-exposure freezing, and extinction freezing). Detailed analyses revealed that vapor history and viral status had minimal influence on the relationships between these behavioral factors of negative affect, suggesting poor interdependence between components of negative affective-like states (see the Supplementary material and Supplemental Table 2 for more comprehensive analyses).

\section{Discussion}

The current study replicates our previous findings that AIE history and stress in adulthood produce sex-specific behavioral outcomes in preclinical tasks that characterize negative affective-like states and are related to BNST dysregulation (summarized in Table 1). In female mice, AIE history and restraint stress increased latency to consume an appetitive reinforcer, indicating an enhanced anhedonic-like state that was independently increased by BNST-mGlu5 receptor knockdown. This notable finding points to specific effects of BNST mGlu5 signaling in regulating anhedonic behavior in female mice. Female mice with mGlu5 knockdown also displayed persistent freezing at the extinction time point compared to viral control mice in a manner that was not modulated by AIE. In males, AIE history enhanced fear acquisition within the training session but mGlu5 knockdown did not alter this response. However, mGlu5 knockdown did increase basal anxiety levels in male mice.

\section{AIE produces distinct behavioral profiles mediated by BNST-mGlu5 receptors}

Previous work using the NIH task has indicated that AIE history enhances latency to consume an appetitive reinforcer in female mice, but only following restraint stress $^{20}$. In the current work, BNST-mGlu5 receptor knockdown increased the latency to consume on the NIH test day independent of AIE history in female mice but did not affect this behavior in male mice. This finding suggests that mGlu5 receptor signaling is required for maintaining baseline behavior, specifically in female mice, and that mGlu5 dysregulation increases anhedonic-like behavior regardless of prior insults. Interestingly, adult female mice with an AIE history show enhanced group 1 mGlu-mediated long-term depression (LTD) in the BNST following restraint stress, while male mice do not. This enhanced LTD is partially mediated by mGlu 5 receptors $^{20}$. In this previous work, overall mGlu5 levels were not altered by AIE in male or female mice ${ }^{20}$. However, these measurements were not able to determine whether 


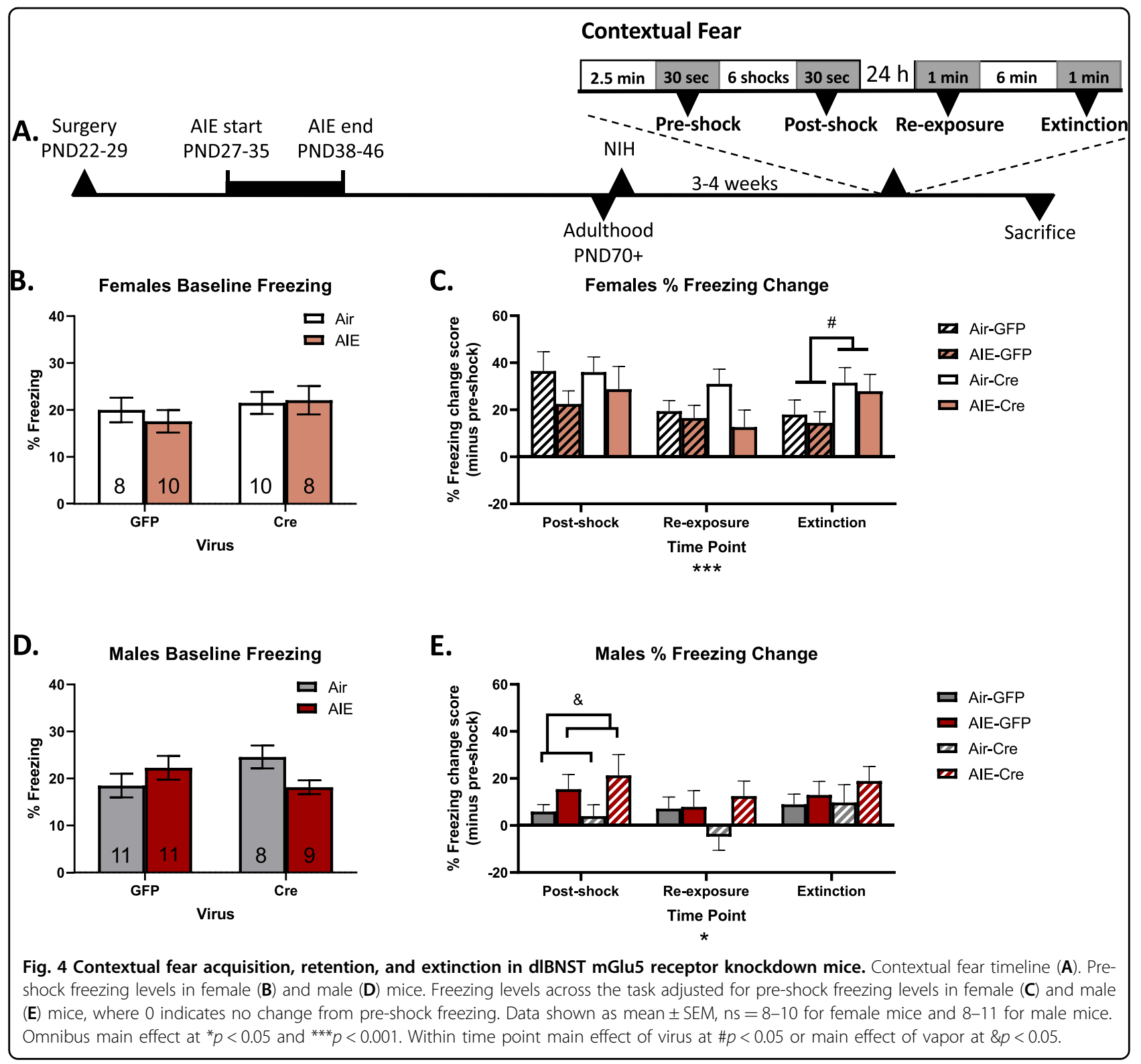

the levels of mGlu5 receptors expressed in the plasma membrane were reduced. Alternatively, BNST-mGlu5 receptor knockdown could upregulate compensatory signaling (e.g., mGlu1 receptors or endocannabinoid signaling). Although the current studies are unable to differentiate between these two possibilities, they highlight the important role that mGlu5 signaling plays in regulating negative affect-like states in female mice. Further, it is possible that the results of these studies may have been different if the mGlu5 receptor knockdown surgery occurred after AIE treatment during late adolescence/ early adulthood instead of prior to AIE during early adolescence. Due to the emergence of negative affectivelike behaviors during adolescence ${ }^{4}$, these studies focused on concurrent mGlu5 receptor knockdown and vapor exposure during adolescent development to better capture relevant changes in mGlu5 signaling that may occur through the entirety of adolescence and play a role in alcohol-induced negative affect ${ }^{26}$.

In adult male mice, restraint stress enhances GluN2Bmediated signaling in the BNST of mice with an AIE history $^{25}$. BNST-GluN2B receptors have been directly implicated in increased consumption latencies in the $\mathrm{NIH}$ task in male mice ${ }^{45}$. These results suggest that sexually divergent mechanisms identified in the BNST are directly related to distinct behavioral outcomes. Group $1 \mathrm{mGlu}$ receptor regional expression levels or intracellular signaling cascades have been historically studied in male rodents. Sex differences in these signaling cascades have not been evaluated in the BNST. One aspect where known 
Table 1 Summary of findings.

\begin{tabular}{|c|c|c|c|c|c|}
\hline & NIH test latency & \multicolumn{2}{|l|}{ Open field test } & \multicolumn{2}{|c|}{ Fear conditioning } \\
\hline & AIE & Air & AIE & Air & AIE \\
\hline \multicolumn{6}{|c|}{ Female mice } \\
\hline GFP & \multirow{2}{*}{$\begin{array}{l}\text { Increased latency } \\
\text { in AIE compared } \\
\text { to air \#, and } \\
\text { increased latency } \\
\text { in Cre } \\
\text { compared to GFP }\end{array}$} & \multirow{2}{*}{\multicolumn{2}{|c|}{ No change in \% center distance for vapor history*viral status }} & (Control) & No change from control \\
\hline Cre & & & & \multicolumn{2}{|c|}{$\begin{array}{l}\text { mGlu5 knockdown enhanced freezing during } \\
\text { extinction regardless of vapor treatment }\end{array}$} \\
\hline \multicolumn{6}{|c|}{ Male mice } \\
\hline GFP & $\begin{array}{l}\text { No change in } \mathrm{NIH} \\
\text { latency for vapor }\end{array}$ & (Control) & $\begin{array}{l}\text { No change } \\
\text { from control }\end{array}$ & (Control) & $\begin{array}{l}\text { Enhanced fear acquisition \# } \\
\text { regardless of viral status }\end{array}$ \\
\hline cre & history*viral status & $\begin{array}{l}\text { mGlu5 knockdown decreased \% Center Distance } \\
\text { (increased negative affective-like behavior) }\end{array}$ & $\begin{array}{l}\text { No change } \\
\text { from control }\end{array}$ & $\begin{array}{l}\text { No change } \\
\text { from control }\end{array}$ & \\
\hline
\end{tabular}

Summary of experimental findings. The pound sign (\#) indicates replications of previous work.

sex differences do exist is in convergence of estrogen and mGlu5 receptor intracellular signaling, whereby mGlu5 signaling can mediate estradiol-driven potentiation of psychostimulant-induced behaviors in female rodents (reviewed in ref. ${ }^{52}$ ).

These distinct mechanisms are further supported by the subthreshold contextual fear results in males. This paradigm was used as a model of hypervigilance ${ }^{48}$, a facet of emotional behavior that is regulated by the BNST in humans and animals ${ }^{21,50,51,53,54}$. Although we observed increased post-shock freezing in AIE-exposed male mice, there was no influence of mGlu5 receptor knockdown on this behavior. As observed previously ${ }^{20}$, AIE history did not significantly alter fear acquisition within the training session in female mice. However, BNST-mGlu5 receptor knockdown did increase freezing in female mice at the extinction timepoint. It is unclear whether this represents persistent fear or is indicative of acclimation to the chamber. The latter appears to be the case in AIE-cre female mice, which showed relatively low levels of freezing at the re-exposure timepoint. This male-specific enhanced fear acquisition within the training session may also be indicative of increased BNST-GluN2B-mediated signaling ${ }^{25}$. Fyn kinase, which regulates GluN2B phosphorylation, has been shown to mediate contextual fear conditioning ${ }^{55-57}$. Previous work has indicated that high alcohol-preferring mice exposed to cued fear conditioning and allowed free access to alcohol show enhanced hypervigilance in the fear-potentiated startle task, regardless of sex. However, fear conditioning only caused immediate increases in alcohol intake in male mice ${ }^{58}$. Another model using unpredictable chronic mild stress showed that an alcohol history plus stress increased binge drinking in male, but not female, mice ${ }^{59}$. Although these studies fundamentally differ from the current model, they may suggest that the hypervigilant state in males driven by adolescent alcohol exposure may fuel increased alcohol intake in adulthood.

Sex-dependent effects were also observed in the open field, where BNST-mGlu5 knockdown increased anxietylike behavior in air-cre, but not AIE-cre, mice. Previous work observing the effects of adolescent alcohol exposure on anxiety indices in adulthood has shown contradictory effects $^{10-12,20}$. Further, the handful of studies observing the role of mGlu5 receptors in adult anxiety-like activity following adolescent alcohol exposure have not demonstrated clear effects of intervention ${ }^{12,60}$. It could be speculated that BNST-mGlu5 knockdown reduced gating of a male-specific neurocircuit involved in the expression of anxiety-like activity. For example, the kappa opioidmediated basolateral amygdala to BNST projection regulates the unconditioned anxiety-like activity and mediates excitatory activity in the $\mathrm{BNST}^{61}$. Whether such circuits are male-specific and mediated by alcohol exposure is unknown.

These sex-specific enhancements of negative affectlike behavior in mGlu5 knockdown mice are surprising given the role of mGlu5 antagonists and negative allosteric modulators in treating depression- and anxiety-like behavior in rodents, as well as the observed behavioral phenotypes of global mGlu5 knockout (KO) animal models. There are numerous possible explanations for the discrepancy between the current results and this literature. First, mGlu5 receptors in the current study are not abolished but are closer to a $60 \%$ reduction 
(see Fig. 1B). There is evidence in some studies that mGlu5 heterozygous (HET) and KO mice show divergent phenotypes from their wild-type counterparts. For example, HET mice show an enhancement of operant sensation seeking, whereas $\mathrm{KO}$ mice show a reduction in their behavioral response ${ }^{62}$. The mice in the current study may be more similar to the HET mice receptor levels versus complete $\mathrm{KO}$. However, divergence in $\mathrm{HET} / \mathrm{KO}$ phenotypes has not generally been seen in studies of generalized anxiety, although to our knowledge NIH has not been assessed. The second potential explanation could be due to the site- and circuit-level roles of the BNST in anxiety and anhedonic behaviors. Optogenetic studies have shown that the behavioral valence outcome of BNST modulation depends on the subnuclei activated $^{63}$. While Cre expression in this study (and thus mGlu5 receptor knockdown) does not seem to be confined to a particular subnuclei, it is possible that mGlu 5 receptor gating of excitatory transmission in this region may have divergent actions. Alternatively, mGlu5 knockdown throughout the BNST may change the activity of specific circuits involved in negative affect-like behaviors. The BNST is a highly interconnected brain region, with reciprocal connections to other regions known to be involved in negative affect, including the cortex, amygdala, and ventral tegmental area ${ }^{64-66}$. mGlu5 receptor knockdown may disrupt LTD in the BNST, particularly in female mice following stress ${ }^{20,67,68}$. Disrupted LTD in the BNST could disturb the balance of excitatory and inhibitory transmission throughout these circuits, resulting in disrupted behavioral outcomes.

\section{Translational relevance}

These findings have robust clinical relevance for investigating underlying mechanisms of the sexually dimorphic risks associated with problematic alcohol use. The NIH task is a model of anhedonia, a classic symptom of depression ${ }^{44}$. Notably, depressive symptoms are stronger drivers of initial and continued alcohol use in young girls and women compared to young boys and men $^{8}$. A recent Phase II clinical trial reported that the mGlu5 negative allosteric modulator basimglurant did not significantly reduce clinician-rated symptoms of MDD in a large sample of individuals without recent alcohol abuse. However, the high dose group (69.4\% female) showed significant improvements in self-reported symptoms of depression $^{36}$. In addition, recent genomewide association studies consistently identify GRM5 as a significantly changed gene in patients with $\mathrm{MDD}^{69-71}$. Paired with the current findings, these results suggest that mGluR5 receptors present an encouraging druggable target and that targeting mGlu5 receptors may alleviate depressive states in women that could proceed problematic alcohol use.
Freezing to low-intensity, unpredictable foot shocks is indicative of a hypervigilant state ${ }^{49}$. Hypervigilance is a distinct facet of the hyperarousal criteria in posttraumatic stress disorder (PTSD) ${ }^{72}$. Although hypervigilance is characteristic of many disorders, like PTSD and generalized anxiety, it is specifically enhanced by unpredictable aversive events in individuals with $\mathrm{PTSD}^{73}$. Within PTSD, hypervigilance is a strong predictor of experiencing other PTSD-related outcomes ${ }^{74}$, which may be associated with problematic alcohol use. Women are more likely to be diagnosed with PTSD than men, but men may be more likely to present with a comorbid $\mathrm{AUD}^{75}$. In predominantly male veteran populations with comorbid AUD, hyperarousal is associated with intrinsic drinking motives and poor adherence to an online PTSD treatment program ${ }^{76,77}$. Although comorbid PTSD and AUD are strongly associated with emotional regulation facets of PTSD in women, this does not include hyper$\operatorname{arousal}^{78}$, similar to the findings in our preclinical model. Our findings may also indicate that adolescent alcohol exposure heightens the risk of developing PTSD-related symptoms.

\section{Conclusion}

Preclinical models with high face validity are necessary to develop treatments for AUDs and comorbid disorders. Although many studies have found that reducing mGlu5 activity alleviates alcohol-induced negative affective states, these studies have focused on males and have not widely included models of dependence. The current findings indicate that AIE produces sexually divergent behavioral phenotypes in adulthood that are unmasked by stress and reflect the human condition. The underlying symptoms and mechanisms of these factors also appear to be sexually divergent, with mGlu 5 receptors playing a large role in depression-related risks in female mice. Notably, these behavioral facets showed poor interdependency, which is further indicative of distinct mechanisms. Overall, these findings support the use of mGlu5 receptors as a treatment target to alleviate negative affective states that may precipitate problematic drinking behavior.

\section{Acknowledgements}

We would like to thank Benjamin Litchfield and Logan Palliser for their assistance in scoring videos. We would also like to thank Dr. Anis Contractor for generating the B6.129-Grm5tm.1.1. Jixu/J mice. This work was supported by the National Institute on Alcohol Abuse and Alcoholism (K99/R00 AA022651 and T32 AA007577) and LSUHSC REP funding.

\section{Author contributions}

C.R.K.: conceptualization, methodology, data collection, data analysis, writingoriginal draft preparation, writing - review and editing, visualization, supervision, project administration; E.B.H.: writing —-review and editing; M.R.L.: data collection, data analysis, validation, writing - review and editing; T.A.W.: conceptualization, writing - review and editing, supervision, funding acquisition. 


\section{Conflict of interest}

The authors declare no competing interests.

\section{Publisher's note}

Springer Nature remains neutral with regard to jurisdictional claims in published maps and institutional affiliations.

Supplementary information The online version contains supplementary material available at https://doi.org/10.1038/s41398-021-01285-y.

Received: 3 July 2020 Revised: 21 January 2021 Accepted: 18 February 2021 Published online: 17 March 2021

\section{References}

1. Nixon, K. \& McClain, J. A. Adolescence as a critical window for developing an alcohol use disorder: current findings in neuroscience. Curr. Opin. Psychiatry 23, 227-232 (2010).

2. Ehlers, C. L. \& Criado, J. R. Adolescent ethanol exposure: does it produce longlasting electrophysiological effects? Alcohol 44, 27-37 (2010).

3. Becker, H. C. Alcohol dependence, withdrawal, and relapse. Alcohol Res. Health 31, 348-361 (2008).

4. Craske, M. G. et al. Anxiety disorders. Nat. Rev. Dis. Prim. 3, 17024 (2017).

5. Rubinow, D. R. \& Schmidt, P. J. Sex differences and the neurobiology of affective disorders. Neuropsychopharmacology 44, 111-128 (2019).

6. Brown, T. A. \& Barlow, D. H. A proposal for a dimensional classification system based on the shared features of the DSM-IV anxiety and mood disorders: implications for assessment and treatment. Psychol. Assess. 21, 256-271 (2009).

7. Koob, G. F. \& Volkow, N. D. Neurocircuitry of addiction. Neuropsychopharmacology 35, 217-238 (2010).

8. Peltier, M. R. et al. Sex differences in stress-related alcohol use. Neurobiol. Stress. 10, 100149 (2019)

9. Crews, F. T. et al. Mechanisms of persistent neurobiological changes following adolescent alcohol exposure: NADIA Consortium Findings. Alcohol Clin. Exp. Res. 43, 1806-1822 (2019).

10. Ehlers, C. L., Liu, W., Wills, D. N. \& Crews, F. T. Periadolescent ethanol vapor exposure persistently reduces measures of hippocampal neurogenesis that are associated with behavioral outcomes in adulthood. Neuroscience $\mathbf{2 4 4}$ $1-15$ (2013)

11. Ehlers, C. L., Criado, J. R., Wills, D. N., Liu, W. \& Crews, F. T. Periadolescent ethano exposure reduces adult forebrain ChAT+IR neurons: correlation with behavioral pathology. Neuroscience 199, 333-345 (2011).

12. Lee, K. M. et al. mGlu5 receptor blockade within the nucleus accumbens shell reduces behavioral indices of alcohol withdrawal-induced anxiety in mice. Front Pharmacol. 9, 1306 (2018).

13. Lee, K. M., Coehlo, M. A., Solton, N. R. \& Szumlinski, K. K. Negative affect and excessive alcohol intake incubate during protracted withdrawal from bingedrinking in adolescent, but not adult, mice. Front Psychol. 8, 1128 (2017).

14. Rico-Barrio, I. et al. Cognitive and neurobehavioral benefits of an enriched environment on young adult mice after chronic ethanol consumption during adolescence. Addict Biol. https://doi.org/10.1111/adb.12667 (2018).

15. Szumlinski, K. K. et al. DID it or DIDn't it? Exploration of a failure to replicate binge-like alcohol-drinking in C57BL/6J mice. Pharm. Biochem. Behav. 178, 3-18 (2019)

16. Van Waes, V. et al. Impact of early life stress on alcohol consumption and on the short- and long-term responses to alcohol in adolescent female rats. Behav. Brain Res. 221, 43-49 (2011).

17. Wright, J. S. \& Panksepp, J. Toward affective circuit-based preclinical models of depression: sensitizing dorsal PAG arousal leads to sustained suppression of positive affect in rats. Neurosci. Biobehav. Rev. 35, 1902-1915 (2011).

18. Kirlic, N. et al. Latent variable analysis of negative affect and its contributions to neural responses during shock anticipation. Neuropsychopharmacology 44 695-702 (2019).

19. Goodwill, H. L. et al. Early life stress leads to sex differences in development of depressive-like outcomes in a mouse model. Neuropsychopharmacology $\mathbf{4 4}$ 711-720 (2019).

20. Kasten, C. R. et al. Adolescent alcohol exposure produces sex differences in negative affect-like behavior and group I mGluR BNST plasticity. Neuropsychopharmacology 45, 1306-1315 (2020).
21. Lebow, M. A. \& Chen, A. Overshadowed by the amygdala: the bed nucleus of the stria terminalis emerges as key to psychiatric disorders. Mol. Psychiatry 21, 450-463 (2016)

22. Kash, T. L, Baucum, A. J. 2nd, Conrad, K. L, Colbran, R. J. \& Winder, D. G. Alcohol exposure alters NMDAR function in the bed nucleus of the stria terminalis. Neuropsychopharmacology 34, 2420-2429 (2009).

23. Wills, T. A. et al. GluN2B subunit deletion reveals key role in acute and chronic ethanol sensitivity of glutamate synapses in bed nucleus of the stria terminalis. Proc. Natl Acad. Sci. USA 109, E278-E287 (2012).

24. Conrad, K. L., Louderback, K. M., Gessner, C. P. \& Winder, D. G. Stress-induced alterations in anxiety-like behavior and adaptations in plasticity in the bed nucleus of the stria terminalis. Physiol. Behav. 104, 248-256 (2011).

25. Carzoli, K. L. et al. Regulation of NMDA receptor plasticity in the BNST following adolescent alcohol exposure. Front. Cell Neurosci. 13, 224 (2019).

26. Kasten, C. R., Holmgren, E. B. \& Wills, T. A. Metabotropic glutamate receptor subtype 5 in alcohol-induced negative affect. Brain Sci. 9, 183 (2019).

27. Gray, A. L. et al. Sex differences in glutamate receptor gene expression in major depression and suicide. Mol. Psychiatry 20, 1057-1068 (2015).

28. Chandley, M. J. et al. Elevated gene expression of glutamate receptors in noradrenergic neurons from the locus coeruleus in major depression. Int. J. Neuropsychopharmacol. 17, 1569-1578 (2014).

29. Meyers, J. L. et al. Frequency of alcohol consumption in humans; the role of metabotropic glutamate receptors and downstream signaling pathways. Transl. Psychiatry 5, e586-e586 (2015).

30. Leurquin-Sterk, G. et al. Cerebral dopaminergic and glutamatergic transmission relate to different subjective responses of acute alcohol intake: an in vivo multimodal imaging study. Addict. Biol. 23, 931-944 (2018).

31. Leurquin-Sterk, G. et al. Lower limbic metabotropic glutamate receptor 5 availability in alcohol dependence. J. Nucl. Med. 59, 682-690 (2018).

32. Ceccarini, J. et al. Recovery of decreased metabotropic glutamate receptor 5 availability in abstinent alcohol-dependent patients. J. Nucl. Med. 61, 256-262 (2020).

33. Akkus, F. et al. Metabotropic glutamate receptor 5 binding in male patients with alcohol use disorder. Transl. Psychiatry 8, 17 (2018).

34. Zerbib, F. et al. Randomised clinical trial: effects of monotherapy with ADX10059, a mGluR5 inhibitor, on symptoms and reflux events in patients with gastro-oesophageal reflux disease. Aliment Pharm. Ther. 33, 911-921 (2011).

35. AstraZeneca. 6-week study treatment to evaluate the safety and effectiveness of AZD2066 in patients with major depressive disorder. https://clinicaltrials. gov/ct2/show/NCT01145755 (2012).

36. Quiroz, J. A. et al. Efficacy and safety of basimglurant as adjunctive therapy for major depression. JAMA Psychiatry 73, 675 (2016).

37. Haass-Koffler, C. L. et al. Administration of the metabotropic glutamate receptor subtype 5 allosteric modulator GET 73 with alcohol: a translational study in rats and humans. J. Psychopharmacol. 32, 163-173 (2018).

38. Metabotropic Glutamate Receptor-5 (mGlur5) Effects on Reward-Related fMRIBOLD Activation in FHP and FHN - Full Text View - Clinical Trials. gov. https:// clinicaltrials.gov/ct2/show/NCT03341715 (2019).

39. Mavoglurant in Alcohol Drinking - Full Text View - Clinical Trials. gov. https:// clinicaltrials.gov/ct2/show/NCT03327792 (2020).

40. Effect of GET73 on MRS Measures of Central Glutamate and GABA in Individuals with Alcohol Use Disorder - Full Text View - Clinical Trials. gov. https:// clinicaltrials.gov/ct2/show/NCT03418623 (2020).

41. Safety and Pharmacodynamic Study of GET 73 in Alcohol Dependent - Full Text View - Clinical Trials. gov. https://clinicaltrials.gov/ct2/show/NCT01842503 (2019).

42. Guide for the Care and Use of Laboratory Animals. vol. 46. Eighth. (The National Academies Press, 2011)

43. Moore, E. M. \& Boehm II, S. L. Site-specific microinjection of baclofen into the anterior ventral tegmental area reduces binge-like ethanol intake in male C57BL/ 6J mice. Behav. Neurosci. 123, 555-563 (2009).

44. Dulawa, S. C. \& Hen, R. Recent advances in animal models of chronic antidepressant effects: the novelty-induced hypophagia test. Neurosci. Biobehav Rev. 29, 771-783 (2005).

45. Louderback, K. M., Wills, T. S., Muglia, L. J. \& Winder, D. G. Knockdown of BNST GluN2B-containing NMDA receptors mimics the actions of ketamine on novelty-induced hypophagia. Transl. Psychiatry 3, e331 (2013).

46. Heinrichs, S. C. \& Koob, G. F. Application of experimental stressors in laboratory rodents. Curr Protoc. Neurosci. Chapter 8:Unit8.4 (2006). 
47. Shansky, R. M. Sex differences in PTSD resilience and susceptibility: challenges for animal models of fear learning. Neurobiol. Stress. 1, 60-65 (2015).

48. Mohammad, F. et al. Concordance and incongruence in preclinical anxiety models: systematic review and meta-analyses. Neurosci. Biobehav. Rev. 68 , 504-529 (2016).

49. Ganella, D. E. \& Kim, J. H. Y. U. Developmental rodent models of fear and anxiety: from neurobiology to pharmacology. Br. J. Pharmacol. 171, 4556-4574 (2014).

50. Sullivan, G. M. et al. Lesions in the bed nucleus of the stria terminalis disrupt corticosterone and freezing responses elicited by a contextual but not by a specific cue-conditioned fear stimulus. Neuroscience 128, 7-14 (2004).

51. Zimmerman, J. M. \& Maren, S. The bed nucleus of the stria terminalis is required for the expression of contextual but not auditory freezing in rats with basolateral amygdala lesions. Neurobiol. Learn Mem. 95, 199-205 (2011).

52. Gross, K. S. \& Mermelstein, P. G. Estrogen receptor signaling through metabotropic glutamate receptors. Vitam. Horm. 114, 211-232 (2020).

53. Knight, L. K. \& Depue, B. E. New frontiers in anxiety research: the translational potential of the bed nucleus of the stria terminalis. Front. Psychiatry 10, 510 (2019).

54. Somerville, L. H., Whalen, P. J. \& Kelley, W. M. Human bed nucleus of the stria terminalis indexes hypervigilant threat monitoring. Biol. Psychiatry 68, 416-424 (2010).

55. Isosaka, T., Kida, S., Kohno, T., Hattori, K. \& Yuasa, S. Hippocampal Fyn activity regulates extinction of contextual fear. Neuroreport 20, 1461-1465 (2009).

56. Isosaka, T. et al. Activation of Fyn tyrosine kinase in the mouse dorsal hippocampus is essential for contextual fear conditioning. Eur. J. Neurosci. 28 973-981 (2008).

57. Kojima, N., Sakamoto, T., Endo, S. \& Niki, H. Impairment of conditioned freezing to tone, but not to context, in Fyn-transgenic mice: relationship to NMDA receptor subunit 2B function. Eur. J. Neurosci. 21, 1359-1369 (2005).

58. Chester, J. A. \& Weera, M. M. Genetic correlation between alcohol preference and conditioned fear: Exploring a functional relationship. Alcoho/ 58, 127-137 (2017).

59. Quadir, S. G. et al. Complex interactions between the subject factors of biological sex and prior histories of binge-drinking and unpredictable stress influence behavioral sensitivity to alcohol and alcohol intake. Physiol. Behav. 203, 100-112 (2019)

60. Lee, K. M., Coelho, M. A., Class, M. A. \& Szumlinski, K. K. mGlu5-dependent modulation of anxiety during early withdrawal from binge-drinking in adult and adolescent male mice. Drug Alcohol Depend. 184, 1-11 (2018).

61. Crowley, N. A. et al. Dynorphin controls the gain of an amygdalar anxiety circuit. Cell Rep. 14, 2774-2783 (2016).

62. Olsen, C. M., Childs, D. S., Stanwood, G. D. \& Winder, D. G. Operant sensation seeking requires metabotropic glutamate receptor 5 (mGluR5). PLoS ONE $\mathbf{5}$ e15085 (2010).

63. Kim, S. Y. et al. Diverging neural pathways assemble a behavioural state from separable features in anxiety. Nature 496, 219-223 (2013).
64. Vranjkovic, O., Pina, M., Kash, T. L. \& Winder, D. G. The bed nucleus of the stria terminalis in drug-associated behavior and affect: a circuit-based perspective. Neuropharmacology 122, 100-106 (2017).

65. Gungor, N. Z. \& Paré, D. Functional heterogeneity in the bed nucleus of the stria terminalis. J. Neurosci. 36, 8038-8049 (2016).

66. Ch'ng, S., Fu, J., Brown, R. M., McDougall, S. J. \& Lawrence, A. J. The intersection of stress and reward: BNST modulation of aversive and appetitive states. Prog. Neuropsychopharmacol. Biol. Psychiatry 87, 108-125 (2018).

67. Grueter, B. A. et al. Extracellular-signal regulated kinase 1-dependent metabotropic glutamate receptor 5-induced long-term depression in the bed nucleus of the stria terminalis is disrupted by cocaine administration. J. Neurosci. 26, 3210-9 (2006).

68. Grueter, B. A., McElligott, Z. A., Robison, A. J., Mathews, G. C. \& Winder, D. G. In vivo metabotropic glutamate receptor 5 (mGluR5) antagonism prevents cocaine-induced disruption of postsynaptically maintained mGluR5dependent long-term depression. J. Neurosci. 28, 9261-70 (2008).

69. Wray, N. R. et al. Genome-wide association analyses identify 44 risk variants and refine the genetic architecture of major depression. Nat. Genet. 50, 668-681 (2018).

70. Gaspar, H. A. et al. Using genetic drug-target networks to develop new drug hypotheses for major depressive disorder. Transl. Psychiatry 9, 117 (2019).

71. Howard, D. M. et al. Genome-wide meta-analysis of depression identifies 102 independent variants and highlights the importance of the prefrontal brain regions. Nat. Neurosci. 22, 343-352 (2019).

72. Association AP, editor. Diagnostic and Statistical Manual of Mental Disorders: DSM-5. 5th edn. (2013).

73. Hoffart, A., Langkaas, T. F., Øktedalen, T. \& Johnson, S. U. The temporal dynamics of symptoms during exposure therapies of PTSD: a network approach. Eur. J. Psychotraumatol. 10, 1618134 (2019).

74. Grillon, C. et al. Increased anxiety during anticipation of unpredictable aversive stimuli in posttraumatic stress disorder but not in generalized anxiety disorder. Biol. Psychiatry 66, 47-53 (2009).

75. Gilpin, N. W. \& Weiner, J. L. Neurobiology of comorbid post-traumatic stress disorder and alcohol-use disorder. Genes, Brain Behav. 16, 15-43 (2017).

76. Livingston, N. A. et al. Changes in alcohol use, PTSD hyperarousal symptoms, and intervention dropout following veterans' use of VetChange. Addict. Behav. 107, 106401 (2020)

77. McDevitt-Murphy, M. E., Luciano, M. T., Tripp, J. C. \& Eddinger, J. E. Drinking motives and PTSD-related alcohol expectancies among combat veterans. Addict. Behav. 64, 217-222 (2017).

78. Goldstein, B., Bradley, B., Ressler, K. J. \& Powers, A. Associations between posttraumatic stress disorder, emotion dysregulation, and alcohol dependence symptoms among inner city females. J. Clin. Psychol. 73, 319-330 (2017).

79. Paxinos, G. \& Franklin, K. B. J. The Mouse Brain in Stereotaxic Coordinates. 2nd edn. (Academic Press, 2001). 